\title{
Diastolic dysfunction and metabolic control impact in patients with type 2 diabetes without cardiovascular disease
}

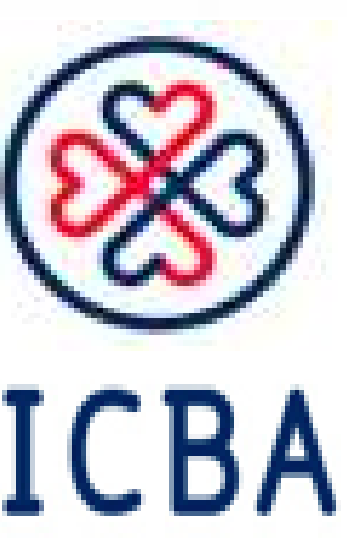

Faingold MC, Chillik I, Vera MI, Ordoñez S, Avegliano G, Ronderos R, Sinay I, Sanabria. H. Instituto Cardiovascular de Buenos Aires (ICBA) Argentina.

\section{Introduction}

Asymptomatic diastolic dysfunction (DD) is common in patients with diabetes (DM). The severity of DD is associated with an increased risk of hospitalization for heart failure and cardiovascular mortality. Although previous trials showed an association between poor metabolic control and the development of DD, many patients included in these studies had established cardiovascular disease or history of previous heart failure, and this makes it difficult to understand the relationship. In our study, we tried to demonstrate the prevalence of DD and its association with metabolic control in a population without previous cardiovascular events or other factors that may act as confounders.

\section{Objectives}

We investigated the association between metabolic control and left ventricular filling pressure in patients with type 2 DM without cardiovascular disease and the correlation of these with the prevalence of DD in this population.

\section{Methods}

We conducted an observational, descriptive, retrospective analysis in 140 patients with a diagnosis of type 2 DM between 18 and 60 years, who consulted the ambulatory diabetes unit care and performed a doppler echocardiogram in these patients. Patients with clinically established cardiovascular disease, previous heart failure, uncontrolled hypertension, moderate to severe valvular disease, atrial fibrillation, creatinine clearance $(\mathrm{Cr})$ less than 60 , anemia, or sleep apnea syndrome were excluded. In addition, three months prior to the study patients had to have their blood pressure under control (blood pressure less than 140/90). We also evaluated the hemoglobin A1C to take into account the metabolic control in the last three months prior to the date of the echocardiogram. The $\mathrm{E} / \mathrm{e}^{\prime}$ ratio was also measured to estimate the left ventricular filling pressure. The association between $\mathrm{HbA} 1 \mathrm{c}$ and $\mathrm{E} / \mathrm{e}^{\prime}$ ratio was analyzed using the Pearlson correlation coefficient, a multivariate linear regression analysis was done to adjust for covariates.

\section{Results}

Clinical characteristics

\begin{tabular}{|l|l|}
\hline Age (mean +/- DS) & 58,6 years $(+/-3,7)$ \\
\hline Men & $60 \%$ \\
\hline HbA1c (mean +/- DS) & $7,2 \%(+/-1,07)$ \\
\hline Time since diagnosis of DM & 5 years $(+/-2)$ \\
\hline
\end{tabular}

Prevalence of DD

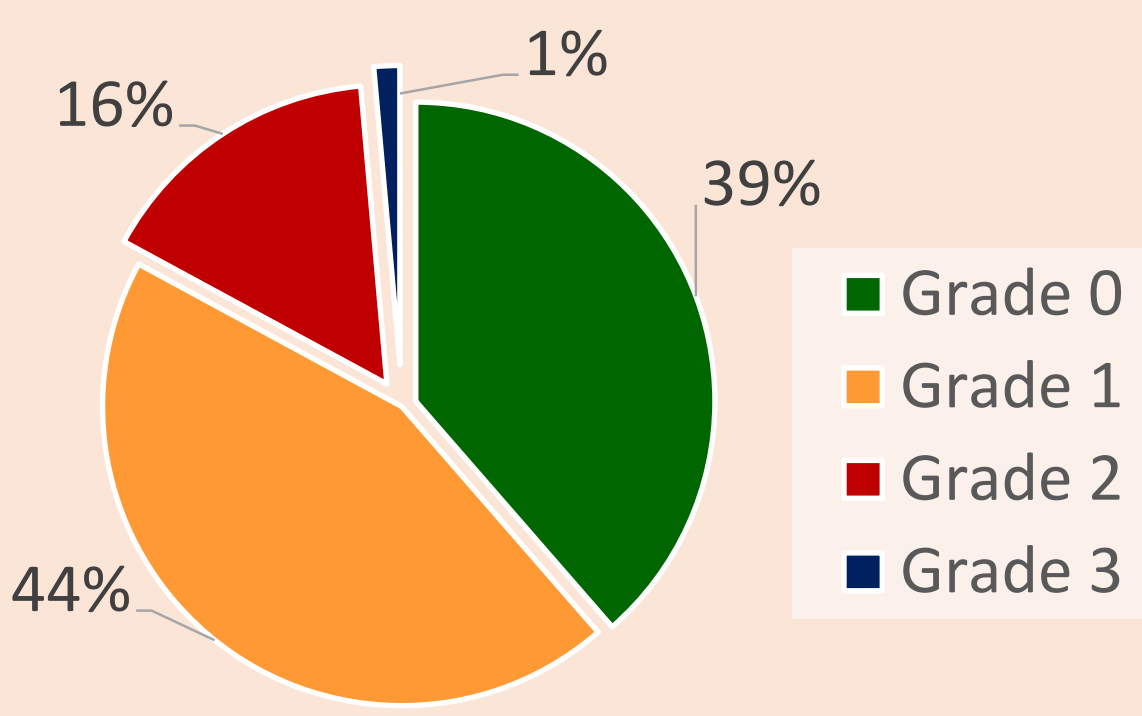

The mean $\mathrm{E} / \mathrm{e}^{\prime}$ ratio was $8.1(+/-2.1)$. We found a significant correlation between the $\mathrm{HbA1C}$ and the $\mathrm{E} / \mathrm{e}^{\prime}$ ratio $(\mathrm{R}=$ $0.836 ; p=0.00001)$, with a $R^{2}$ value of $0.699(p=0.0001)$. The relationship between the two variables was directly proportional $(B=1.649, p=00001)$. In a multivariate regression analysis, by adjusting $\mathrm{HbA1c}$, age, systolic blood pressure, $\mathrm{Cr}$ and hemoglobin; this linear association was maintained.

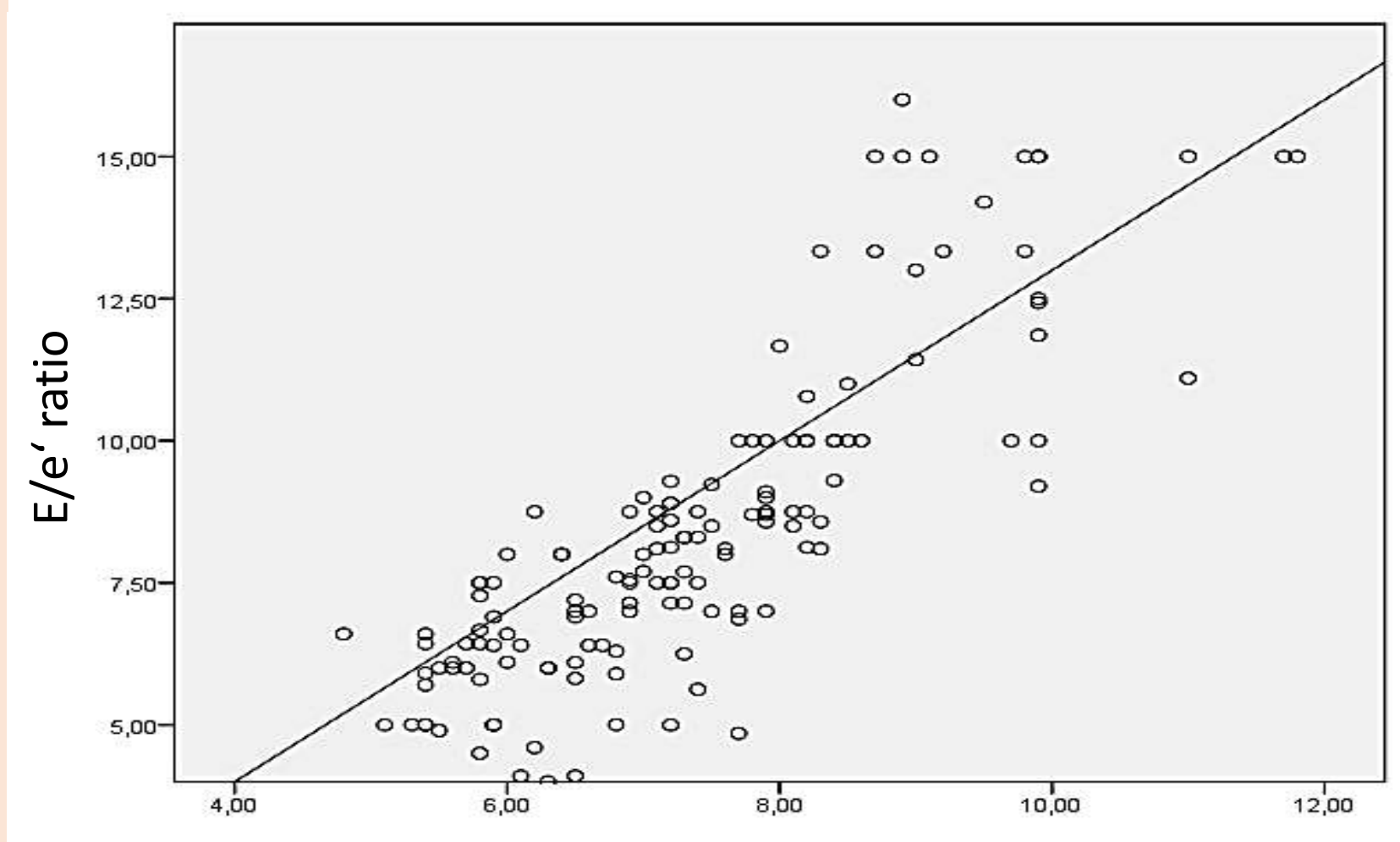

HbA1c

\section{Conclusions}

DM is associated with a high prevalence of moderate to severe diastolic dysfunction in people younger than 60 years without previous cardiovascular events. The poor metabolic control is directly associated with increased left ventricular filling pressures. Our results enhance previous observations and may be relevant at the time of choosing a treatment or establish strategies to prevent the development of heart failure. 